\title{
Scaling hard vertical surfaces with compliant microspine arrays
}

\author{
Alan T. Asbeck, Sangbae Kim, \\ and M.R. Cutkosky \\ School of Engineering \\ Stanford University \\ Stanford, California 94305-2232 \\ Email: aasbeck, sangbae, cutkosky@ stanford.edu
}

\author{
William R. Provancher \\ University of Utah \\ Salt Lake City, Utah 84112-9208 \\ Email: wil@eng.utah.edu
}

\author{
Michele Lanzetta \\ University of Pisa \\ Pisa, Italy \\ Email: michele.lanzetta@ing.unipi.it
}

\begin{abstract}
A new approach for climbing hard vertical surfaces has been developed that allows a robot to scale concrete, stucco, brick and masonry walls without using suction or adhesives. The approach is inspired by the mechanisms observed in some climbing insects and spiders and involves arrays of microspines that catch on surface asperities. The arrays are located on the toes of the robot and consist of a tuned, multi-link compliant suspension. In this paper we discuss the fundamental issues of spine allometric scaling versus surface roughness and the suspension needed to maximize the probability that each spine will find a useable surface irregularity and to distribute climbing tensile and shear loads among many spines. The principles are demonstrated with a new climbing robot that can scale a wide range of exterior walls.
\end{abstract}

\section{INTRODUCTION}

Previously developed climbing robots have generally employed suction cups [19], [20], [29], magnets [5], [27] or sticky adhesives [2] to cling to smooth vertical surfaces such as windows and interior walls. None of these approaches is suitable for porous and typically dusty exterior surfaces such as brick, concrete, stucco or stone. Other robots employ hand and foot holds in the manner of a human climber [6], [8]. A recent innovation employing a controlled vortex [1] to create negative aerodynamic lift has been demonstrated on brick and concrete walls with considerable success. However, this approach consumes significant power (including when the robot is stationary), unavoidably generates noise and is difficult to adapt to non-flat surfaces such as window ledges and corners.

When we look at animals that exhibit scansorial (vertical surface) agility, we find a variety of methods employed [9]. Larger animals such as cats and raccoons employ strong claws that penetrate wood and bark surfaces. Tree frogs and many insects employ sticky pads [12], [13]. Geckos and some spiders employ large numbers of very fine hairs that achieve adhesion via van der Waals forces on almost any kind of surface [4], [18], [3]. Other insects, arthropods and reptiles employ small spines that catch on fine asperities [11]. All of these approaches are worthy of examination for climbing robots. However, dry adhesives and spines are particularly attractive for hard, dusty, exterior surfaces.
Several researchers are currently working on creating synthetic versions of the setae found on geckos or the scopulae seen on spiders [21], [26], [14]. The early results are intriguing but current synthetic adhesives are not able to sustain the kinds of tensile loads needed at the forelimbs of a climbing robot. Moreover, they are fragile and, as yet, lack the self-cleaning property that allows geckos to climb dusty walls.

\section{SPINE AND SURFACE SCALING}

Insects and arthropods that climb well on vertical surfaces often have legs equipped with large numbers of small, sharp spines. At a larger scale, geckos that frequent rock surfaces such as cliffs and caves have small claws on each toe in addition to their dry adhesive structures [28]. Unlike the larger claws of a cat, the tiny spines or claws do not need to penetrate the surface. Instead, they engage small asperities (bumps or pits). Several studies in the biology literature have considered the problem of spine/surface interaction. Dai et al. [11] present a planar model of spine/asperity contact and compute the maximum load per spine as a function of spine strength, relative size of the spine tip versus that of an asperity, and coefficient of friction. As expected, for rough surfaces the mechanical strengths of the spine and asperity become the limiting factors; for smoother surfaces friction is more important and the ability to pull in toward the surface is much reduced.

Given the general correlation in nature between spine or claw size and animal size, we are led to ask: For a climbing robot of a certain size, how large should the spines be? For a $0.4 \mathrm{Kg}$ robot we might expect spines or claws similar to those seen in squirrels or large climbing lizards. However, this argument ignores the point that spines of hardened steel are much stronger and stiffer than their natural counterparts. Indeed, if the strength of the spine/asperity contact were not a constraint, we should make the spines as small as possible. The reason behind this argument is that many natural surfaces, and some man-made surfaces such as concrete and stucco, have an approximately fractal surface topography [10], [15], [16] so that characteristic surface features (asperities) can be found over a wide range of length scales. 


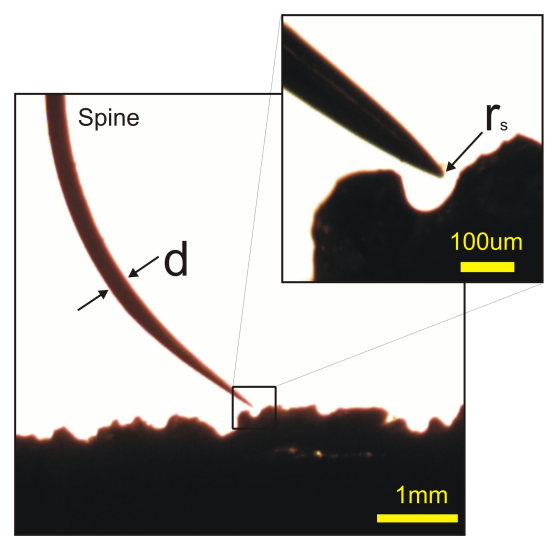

Fig. 1. Profile photograph of typical spine, with shaft dia., $d=270 \mu m$ and tip radius $r_{s}=10 \mu \mathrm{m}$, engaging a rough profile of 80 grit aluminum oxide sandpaper.

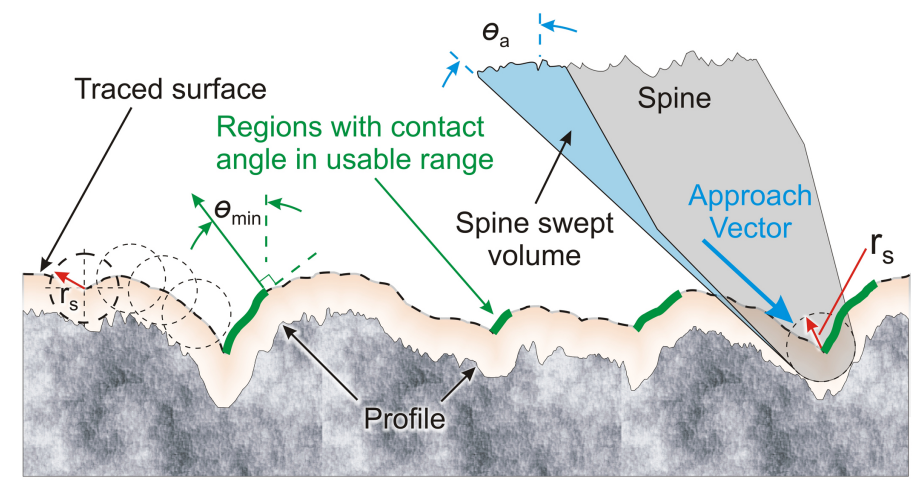

Fig. 2. Diagram of the spine/surface interaction model. Spine approaches from left and creates a swept volume that produces a traced surface with respect to the original profile. Contiguous leftward-sloping regions, starting when $\theta>\theta_{\min }$, are available for sustaining loads.

In the following sections we first investigate the question of spine/asperity scaling in more detail, using surface profiles obtained for various materials - some of which our climbing robot can scale easily and some of which are too smooth for the spines that we currently use. The results of this analysis show that while surfaces do, in general, present more asperities per unit area as spine size shrinks, the details of the spine approach angle and the surface topography also play an important role. We next consider the question of spine/asperity contact strength. There are several possible failure modes. In general, however, the expected maximum load increases as the square of overall spine size.

\section{SPINE/SURFACE MODELLING}

Simulations were done to model the behavior of spines interacting with surfaces, to determine what size spines are needed to climb various surfaces and to understand the properties of a surface that lead to its having good asperities. In general, the simulations fitted 2-D spine cross-sections to 2D surface profiles and determined the locations where the simulated spine could perch on the profile.
Two-dimensional profiles of several stone and sandpaper surfaces were obtained using a profilometer (Taylor Hobson Talysurf Series 2), with a conical stylus terminating in spherical tip of radius $2 \mu \mathrm{m}$. Profiles of rougher concrete surfaces were measured using a laser micrometer (Omron Z4M-N30V). In analyzing these profiles we consider a typical spine as shown in the photograph in Figure 1. The spine is a curved beam with a circular cross section that tapers to a rounded tip of radius $r_{s}$. The spine is attached to a robot limb that drives it toward the surface at an approach angle, $\theta_{a}$, and subsequently strokes it downward (from left to right in Figures 1 and 2), hoping to engage an asperity.

We determine the regions of the surface that a spine could perch on in the following manner. We compute the intersection of the swept volume of the spine with respect to the surface profile. The result of this computation is a traced surface, as shown in Fig. 2. Next we search along the traced surface starting from the right ("downward" end) toward the left in Figure 2. We search for locations at which the traced surface normal, given by the angle $\theta$, is above a minimum useable angle, $\theta_{\text {min }}$. $\theta_{\text {min }}$ depends on the spine loading and coefficient of friction. We consider regions between these locations and the subsequent minimum in the traced surface to be "useable asperities." For a given RMS surface roughness, $R_{q}$, the useable asperity regions can vary greatly, depending on the details of the profile.

\section{A. Simulation results}

Profiles were taken for several stone surfaces and several types of sandpaper, listed in Table I. This table also shows the largest tip radius at which there is a high probability of engaging at least a few asperities per centimeter of stroke, for several values of approach angle, $\theta_{a}$, and assumed minimum value of the surface normal, $\theta_{\text {min }}$. One centimeter corresponds approximately to the travel of the toe mechanism supporting the spines in SpinyBotII (described in Section V).

Figure 3 shows typical results for the number of asperities per centimeter for selected stone and sandpaper surfaces, with constant values of $\theta_{\min }$ and $\theta_{a}$. For the stone surfaces (solid curves) the number of asperities per centimeter is initially observed to decrease at a rate similar to the expected $1 / r$ trend for a fractal surface. Above a certain tip radius, however, the number of available asperities drops rapidly. This is an effect of the cutting, polishing and wearing processes that have partially smoothed the stone surfaces so that large asperities are rare. The distributions of lengths between asperities, and the asperity lengths themselves, are approximately exponential random variables with a probability density function

$$
f_{X}(x)=\lambda * \exp (-\lambda * x), x \geq 0,
$$

where $x$ is the distance between asperities (or the asperity length). The number of asperities per $\mathrm{cm}$ values on the plots are values of the lambda rate parameter; the mean distance between asperities is $1 / \lambda$ and the variance is $1 / \lambda^{2}$.

In comparison to the curves for the stone and concrete, the curves for the sandpaper have a shallow slope of less than $1 / r$ 


\begin{tabular}{|c|c|c|c|c|c|c|c|c|c|c|c|c|}
\hline \multirow[t]{3}{*}{$\begin{array}{l}\text { Surf. } \\
\text { No. }\end{array}$} & \multirow[t]{3}{*}{ Surface } & \multirow{3}{*}{$\begin{array}{c}\text { Average } \\
\text { Roughness } R a \\
(\mu \mathrm{m})\end{array}$} & \multirow{3}{*}{$\begin{array}{c}\text { RMS } \\
\text { Roughness } R q \\
(\mu \mathrm{m})\end{array}$} & \multicolumn{6}{|c|}{$\begin{array}{c}\text { Approach Angle } \theta_{a} \\
45^{\circ}\end{array}$} & \multicolumn{3}{|c|}{$80^{\circ}$} \\
\hline & & & & \multicolumn{3}{|c|}{$\theta_{\min }$} & \multicolumn{3}{|c|}{$\theta_{\min }$} & \multicolumn{3}{|c|}{$\theta_{\min }$} \\
\hline & & & & $45^{\circ}$ & $65^{\circ}$ & $80^{\circ}$ & $45^{\circ}$ & $65^{\circ}$ & $80^{\circ}$ & $45^{\circ}$ & $65^{\circ}$ & $80^{\circ}$ \\
\hline 1 & cobblestone & 56.85 & 78.09 & 52.0 & 33.3 & 20.0 & 52.2 & 33.5 & 20.2 & 41.9 & 26.4 & 12.1 \\
\hline 2 & machined granite & 6.55 & 10.26 & 28.3 & 17.5 & $\mathrm{NaN}$ & 28.4 & 17.6 & $\mathrm{NaN}$ & 19.1 & 9.5 & $\mathrm{NaN}$ \\
\hline 3 & rough cut granite & 53.54 & 67.49 & 56.0 & 40.0 & 27.5 & 56.1 & 40.2 & 27.6 & 45.1 & 35.5 & 20.5 \\
\hline 4 & polished granite & 13.23 & 21.01 & 39.8 & 29.9 & $\mathrm{NaN}$ & 38.3 & 29.9 & $\mathrm{NaN}$ & 28.0 & 21.0 & $\mathrm{NaN}$ \\
\hline 5 & paving stone & 73.03 & 92.16 & 70.0 & 40.0 & 14.4 & 70.2 & 40.1 & 14.4 & 61.8 & 37.6 & 13.8 \\
\hline 6 & concrete cinderblock & 92.96 & 131.93 & 282.9 & 162.6 & 101.2 & 283.7 & 163.4 & 101.6 & 126.9 & 73.9 & $\mathrm{NaN}$ \\
\hline 7 & concrete 2 & 70.14 & 88.42 & 159.5 & 139.1 & $\mathrm{NaN}$ & 159.6 & 139.3 & $\mathrm{NaN}$ & 140.3 & 93.5 & $\mathrm{NaN}$ \\
\hline 8 & Al-oxide 80 & 42.37 & 57.89 & 120.1 & 90.0 & 40.0 & 120.5 & 88.2 & 40.3 & 107.6 & 72.9 & 32.8 \\
\hline 9 & Al-oxide 100 & 35.83 & 49.89 & 110.1 & 86.7 & 70.0 & 107.1 & 80.6 & 57.9 & 88.6 & 62.9 & 41.9 \\
\hline 10 & Al-oxide 120 & 20.30 & 26.05 & 68.0 & 48.6 & 15.0 & 68.2 & 48.7 & 25.1 & 55.5 & 32.8 & $\mathrm{NaN}$ \\
\hline 11 & Al-oxide 150 & 21.68 & 27.82 & 60.0 & 55.0 & 43.3 & 60.2 & 53.4 & 40.2 & 55.5 & 33.2 & 24.1 \\
\hline 12 & Painter's 100 & 30.54 & 38.88 & 105.0 & 40.0 & $\mathrm{NaN}$ & 100.4 & 37.6 & $\mathrm{NaN}$ & 95.3 & 38.2 & $\mathrm{NaN}$ \\
\hline
\end{tabular}

\section{TABLE I}

TABLE SHOWING THE ROUGHNESS PARAMETERS AND MAXIMUM SPINE TIP RADIUS FOR ENGAGING ASPERITIES ON VARIOUS STONE AND SANDPAPER SURFACES. THE COLUMNS OF DATA UNDER EACH APPROACH ANGLE INDICATE THE MAXIMUM SPINE TIP RADIUS, IN $\mu$ M, THAT HAS A HIGH PROBABILITY OF ENGAGING AT LEAST A FEW ASPERITIES PER CENTIMETER OF VERTICAL STROKE ALONG THE SURFACE. EACH ENTRY IS FOR A GIVEN

APPROACH ANGLE, $\theta_{a}$ AND ASSUMED VALUE OF $\theta_{\text {min }}$, WHICH DEPENDS ON THE COEFFICIENT OF FRICTION. LARGER NUMBERS INDICATE A GENERALLY ROUGHER SURFACE THAT IS EASIER TO CLIMB WITH LARGER SPINES. A VALUE OF NAN INDICATES THAT THE SURFACE WAS SO SMOOTH THAT THE SIMULATION DID NOT FIND ANY ASPERITIES FOR ANY RADIUS ABOVE $8 \mu \mathrm{M}$.

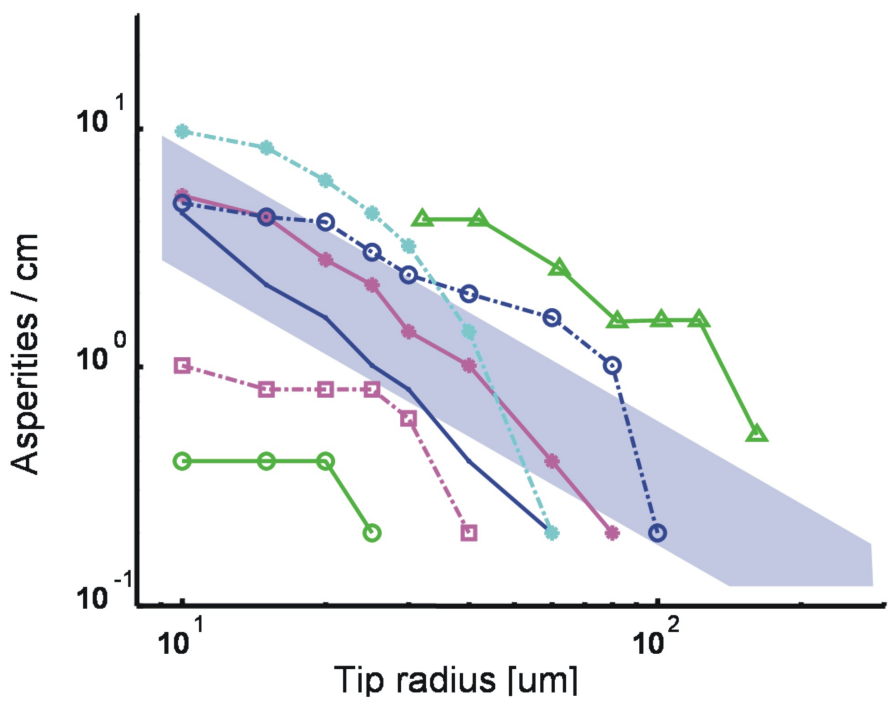

Fig. 3. Number of asperities per cm for various surfaces, with $\theta_{a}=45^{\circ}$ and $\theta_{\min }=75^{\circ}$. Solid lines indicate stone and concrete surfaces, and dashed lines indicate sandpaper surfaces. A band corresponding to the $1 / r$ trend that would be expected for a perfect fractal surface has been added for reference.

for small tip radii. This occurs because the sandpapers have a relatively uniform particle size. For small radii, the 150 and 120 grit aluminum oxide sandpapers have more asperities than the larger-grained 80 and 100 grit aluminum oxide sandpapers, but for large radii the reverse is true. Also, for large assumed values of $\theta_{\min }>65+^{\circ}$, corresponding to a low assumed coefficient of friction, the 100 grit painter's sandpaper has substantially fewer asperities than 100 grit aluminum oxide

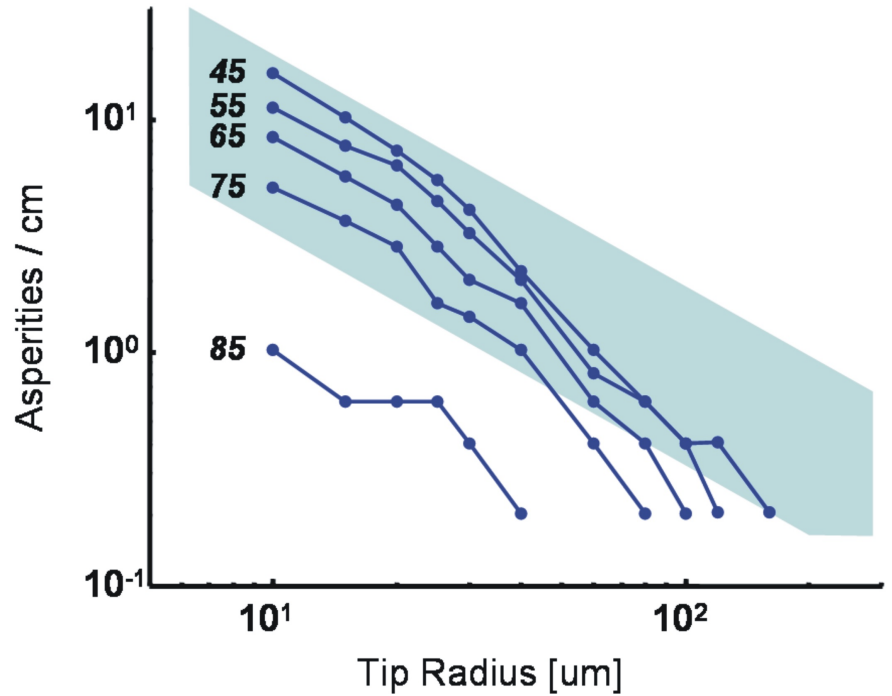

Fig. 4. Number of asperities per $\mathrm{cm}$ as $\theta_{\min }$ is varied, and $\theta_{a}$ is held constant at $65^{\circ}$, for a stone surface. A band corresponding to the $1 / r$ expected for a perfect fractal surface is shown for comparison.

because it has more rounded particles.

Figure 4 shows the number of asperities per centimeter as $\theta_{\text {min }}$ is varied, with constant $\theta_{a}$. As $\theta_{\min }$ increases, we are less likely to find useable asperities. The distribution of traced surface normal angles is approximately Gaussian, dropping to close to 0 at $\theta= \pm 90^{\circ}$. The shape of this distribution is reflected in the various vertical offsets in the figure. As $\theta_{a}$ is varied, with constant $\theta_{\min }$, the number of asperities $/ \mathrm{cm}$ 
changes relatively little, decreasing significantly only for large approach angles where the spine is nearly parallel to the wall. For small approach angles, the spine's ability to reach the regions of the surface it hooks on to is only slightly affected.

\section{B. Correlation with climbing robot performance}

In this section we compare the results of the foregoing analysis to the empirical results obtained with our climbing robot. The parameters $\theta_{\text {min }}$ and $\theta_{a}$ used by our climbing robot, SpinyBotII, were measured and used in the simulation. Based on the geometry of SpinyBotII, the feet are loaded at angles $3.5^{\circ}<\theta_{\text {load }}<8^{\circ}$ degrees from the wall. For a given coefficient of friction, $\mu$, we have:

$$
\theta_{\text {min }}=\theta_{\text {load }}+\operatorname{arccot}(\mu) \text {. }
$$

The coefficient of friction between stainless steel spine tips and rock is generally between 0.15 and 0.25 , which corresponds to $\theta_{\min }$ between $86.5^{\circ}$ and $81^{\circ}$ for an average $\theta_{\text {load }}$ of $5^{\circ}$. The approach angle, $\theta_{a}$, is from $45^{\circ}-65^{\circ}$, based on the angle of the spines themselves and the motion of the tip over the surface. The tip radii, $r_{s}$, of the spines are 10-15 $\mu \mathrm{m}$ for new spines and 25-35 $\mu \mathrm{m}$ for spines dulled due to heavy use. The behavior we see using these values qualitatively corresponds to the actual performance of SpinyBotII on the corresponding surfaces. SpinyBotII works extremely well on the Al-oxide 100 and 80 grit sandpapers, moderately-to-poorly on the Al-oxide 150 and 120 grit, and poorly on the painter's 100 grit sandpaper. On the natural surfaces, SpinyBotII works very well on the rough concretes, moderately well on the rough cut granite, and not at all on the ground and polished surfaces. In the simulation, if $\theta_{\min }$ is $82^{\circ}-85^{\circ}$ and $\theta_{a}$ is $45^{\circ}$ or $65^{\circ}$, at radii of $10-40 \mu \mathrm{m}$ the relative ordering of the surfaces in number of asperities/cm is generally correct, with the 100 grit Al-oxide sandpaper having the most asperities, followed by the 150,80 grit sandpapers and the cut granite block surface.

The concrete profiles show somewhat fewer asperities than would be expected for small tip radii. this is because they were recorded using a laser micrometer with a $65 \mu \mathrm{m}$ spot size that produced some low-pass filtering. Consequently the concrete profiles do not appear in the correct order for simulated asperity densities. In general, though, the ordering of the surfaces appears to be correct. All of the surfaces show fewer asperities than would be expected if $\theta_{\min }$ is increased above around $82^{\circ}$. This discrepancy is likely due to the reduced ability of the measurement instruments to accurately record abrupt changes in surface height.

\section{Discussion}

Dai et al. [11] present a planar model of spine/asperity contact that includes the relative size of the spine tip versus that of an asperity and coefficient of friction. Our analysis is similar, except that we use actual surface contours and compute the corresponding traced surface for the swept volume produced by a spine. Dai et al. state that the angle of spine/surface contact is key to obtaining traction between beetle claws and surfaces. However, they frame their conclusions in terms of general surface $R M S$ roughness $R_{q}$, a different parameter entirely. Our results indicate that the normal angle, $\theta$, is the critical parameter, and that surface roughness measures $R_{a}$ and $R_{q}$ are not always correlated with actual spine performance. As an example, the $R_{a}$ and $R_{q}$ of the painter's 100 grit sandpaper are larger than the corresponding values for 120 and 150 grit Al-oxide, but it has many fewer useable asperities.

Our present analysis is limited in that it cannot detect the presence of angles more than 90 degrees (upward-leaning overhangs). Even at angles close to 90 degrees it is less accurate due to the non-zero cone angle of the profilometer stylus. It may be that mechanical interlocking with angles greater than 90 degrees is the cause of many of the asperities that SpinyBotII actually uses.

Also, the observed behavior of spines interacting with surfaces differs slightly from that assumed by the model. As a foot is brought into contact with surface and begins its downward stroke, we observe that many spines briefly catch on "pseudo-asperities" and then break away as the load is ramped up, possibly due to slight spine deflections, which lead to spine slipping. If the load were kept small these "pseudo-asperities" would probably be useable. The spines also tend to skip over the surface (i.e., to become briefly airborne) after slipping off the "pseudo-asperities" and they undergo alternating regimes of static and dynamic friction. The effective coefficient of friction while this occurs is probably quite low. Finally, there is a chance that as a compliant spine drags down the wall it may tend to follow a local groove or valley and thereby be steered away from protruding asperities. Conversely, negative asperities (pits) will tend to steer the spine into a favorable location for obtaining a grip. Hence, actual spine/surface dynamics vary depending on the surface type as well as how much the spine is able to move in the direction perpendicular to its travel.

\section{SPINE/ASPERITY CONTACT STRENGTH}

While smaller spines are more effective at engaging asperities on smooth surfaces, they also carry smaller loads. We have found that when steel spines catch on asperities on concrete or stucco, the contact typically fails in one of three ways:

- plastic failure of the base of the spine in bending,

- excessive elastic rotation of the spine tip causing it to slip off the asperity,

- brittle failure of the asperity itself.

In each of these cases, if we take a dimension such as the spine tip radius, $r_{s}$, as a characteristic length and scale everything uniformly, then the maximum load of the spine/asperity contact increases as $r_{s}^{2}$ (see Appendix for details).

Figure 5 shows graphically how the maximum load of the spine/asperity contact increases as $r^{2}$, while the expected number of asperities per unit area decreases as $1 / r^{2}$. Measurements of contact strength were done using spines of various sizes on concrete and sandpaper samples attached to a load cell. We note that the sandpaper consisted primarily of male features (which had a small bonding cross-section). Therefore, asperity failure tended to occur before spine failure on that surface. In 


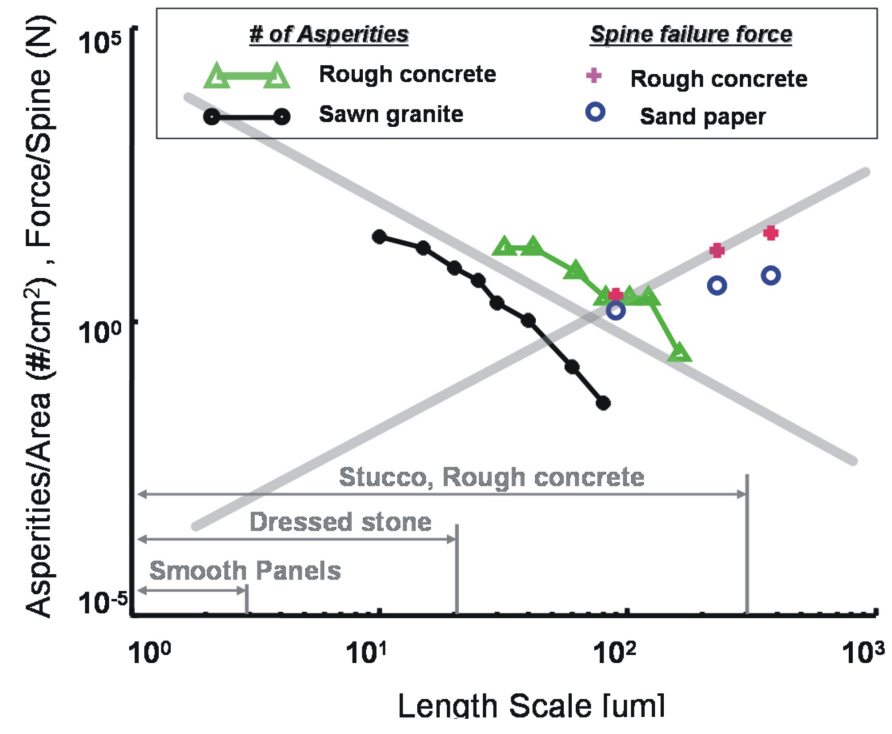

Fig. 5. Log/log plot showing the expected $r^{2}$ trend of spine/asperity contact strength versus the expected $1 / r^{2}$ trend for asperities per unit area of the wall. The data for surfaces of rough cut granite and concrete are plotted for comparison with the expected asperity density trend. Individual tests of spine/asperity failures are plotted for concrete and sandpaper surfaces.

contrast, the cast concrete primarily consisted of female features that were consequently much stronger; so spine failure, either by excessive end rotation or plastic bending, tended to dominate. For other surfaces, all three failure modes tended to occur simultaneously. The figure also shows the asperity density data for a concrete and machined granite surface as compared to the expected $1 / r^{2}$ for fractal surfaces. At the bottom of the figure, the representative asperity length scales for a few different surfaces are indicated. These indicate, for example, that rough concrete surfaces will present useable asperities of up to $300 \mu \mathrm{m}$ whereas smooth concrete or stone panels will present asperities of up to $20 \mu \mathrm{m}$. These values essentially impose an upper limit to the spine size that can be used with these surfaces.

For our first climbing robot, SpinybotI, we employed 4 spines per foot, each with a tip radius of approximately $30 \mu \mathrm{m}$. This machine was able to climb stucco and rough concrete reliably. The spine/asperity contacts could sustain loads of several $\mathrm{N}$, usually limited by brittle failure of the asperity rather than of the spine. However, for surfaces such as smooth concrete and dressed stone, the probability of a spine encountering a useful asperity during a vertical stroke length of approximately $2 \mathrm{~cm}$ was too low for reliable climbing. SpinybotII employs two rows of spines on each foot, each spine having a tip radius of approximately $15 \mu \mathrm{m}$. The maximum force per spine/asperity contact is $1-2 \mathrm{~N}$, and the probability of finding useable asperities per centimeter of stroke length is high.

To summarize the preceding discussion, as spines become smaller we can ascend smoother surfaces because the density of useable spine/asperity contacts increases rapidly. However, we need larger numbers of spines because each contact can

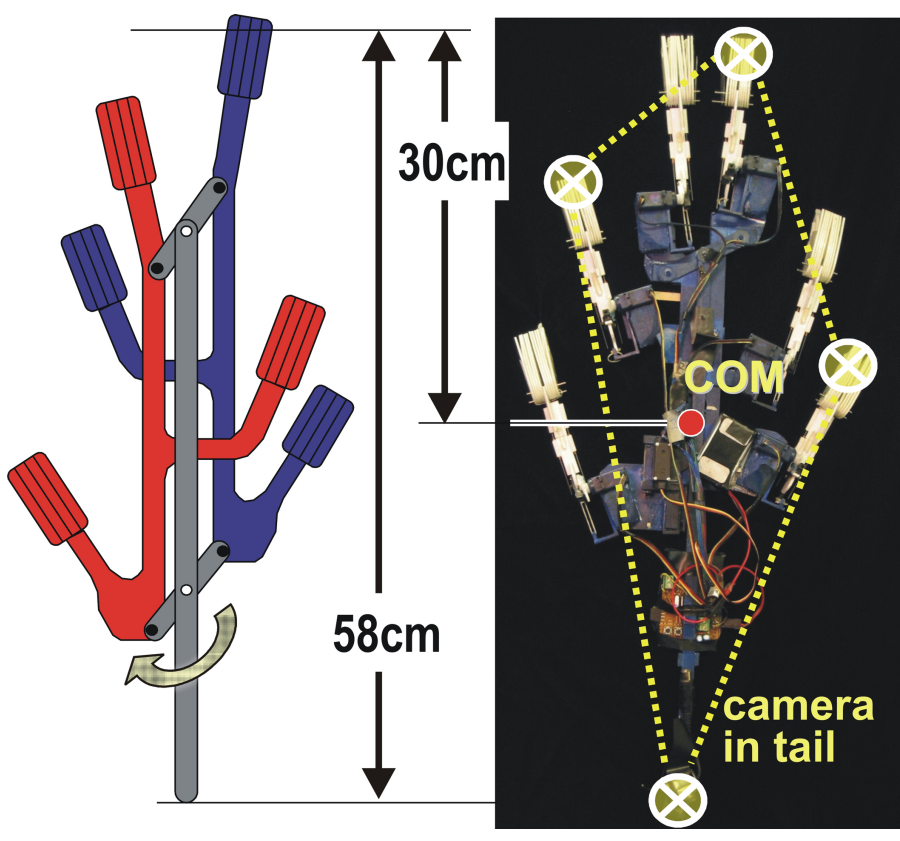

Fig. 6. Photograph of SpinyBotII on wall and diagram of climbing mechanism. Each set of three legs is attached to a mechanism such that the robot employs a fixed alternating-tripod gait. A long tail helps to reduce the pitching moment. The center of mass (COM) is always within a polygon of contacts, to minimize yawing rotations in the plane of the wall.

sustain less force. In order to make use of large numbers of spines, the two key design principles are:

- ensure that as many spines as possible will independently find asperities to attach to and

- ensure that the total load is distributed among the spines as uniformly as possible.

In the next section we briefly describe the design of SpinybotII that embodies these design principles.

\section{SPINYBOTII: CLIMBING WITH COMPLIANT MICROSPINES}

\section{A. Body Design: Promoting Load Sharing and Stability}

Figure 6 shows a plan view schematic and photograph of SpinybotII. The robot uses an alternating tripod gait, as found in climbing insects. At any time, the robot is clinging by three feet. Like many climbing animals, the robot also has a tail which reduces the "pull-in" forces needed at the front limbs to overcome the pitching moment produced by gravity acting at the center of mass, which is located approximately $2 \mathrm{~cm}$ outward from the wall. The total weight of the robot, including lithium polymer batteries, wireless camera, and PIC microprocessor is $0.4 \mathrm{Kg}$. It can carry an additional payload of $0.4 \mathrm{Kg}$ while climbing. The climbing speed is currently quite slow $(2.3 \mathrm{~cm} / \mathrm{s})$ but can easily be increased by using a more powerful motor for the alternating tripod mechanism. While the main concern for vertical climbing is to avoid pitching back from the plane of the wall, it is also important to maintain rotational stability in the plane of the wall. As seen in Fig. 6 , the center of mass of SpinybotII lies within a polygon of 
contacts at all times. Also, as observed in climbing insects and reptiles, the legs have a slight inward pull, toward the centerline of the robot. This arrangement reduces the upsetting moments (in the plane of the wall) about the center of mass, should one of the legs momentarily lose its grip.

\section{B. Toe and Foot Design: Promoting Spine Attachment and Load Sharing}

The feet on SpinybotII represent the sixth generation of a compliant, spined design. A failing of earlier designs was that on close observation, only a few spines were carrying most of the load. Each foot of SpinybotII contains a set of 10 identical planar mechanisms, or "toes." The mechanisms are created using a rapid prototyping process, Shape Deposition Manufacturing (SDM) [22], [7] that permits hard and soft materials to be combined into a single structure. Figure 7 shows a side vide of the robot on a concrete wall and a detail of a single foot, showing several of the planar toe mechanisms, each of of which bears two spines (several of which are visibly engaged).

Each of the toes is a compliant multi-bar linkage, independent of its neighbors and able to stretch parallel to the wall under a load. Thus, if a toe catches an asperity, neighboring toes are not prevented from catching their own asperities because they will continue to slide down the wall as the caught toe stretches. The grey material in the photograph is a soft urethane (Innovative Polymers Inc.) of 20 Shore-A hardness and the white material is a hard urethane of 75 Shore-D hardness. The spines are approximately $1.5 \mathrm{~mm}$ long with a $200 \mu \mathrm{m}$ shaft diameter and $15 \mu \mathrm{m}$ tip radius. They are embedded directly into the hard white links during the SDM process. The soft urethane flexures provide both elasticity and viscoelastic damping. They permit greater extensions without failure than miniature steel springs (as were used on some of the earlier foot designs). The distal flexures are designed to buckle slightly so that the toe is very compliant with respect to positive normal forces (i.e. forces pushing outward from the wall) but stiffer when loaded in tension. In addition, the linkage is designed so that an initial contact at the inner (proximal) spine forces the distal spine outward to facilitate engagement. Finally, it is designed so that tensile loading does not cause the spines to rotate upward, which would make them tend to slip off asperities that they have engaged.

\section{CONCLUSIONS AND FUTURE WORK}

\section{A. Conclusions}

SpinybotII climbs reliably on a wide variety of hard, outdoor surfaces including concrete, stucco, brick, and dressed sandstone with average asperity radii of greater than about $25 \mu \mathrm{m}$. Scaling SpinyBotII to larger payloads is straightforward; one simply needs more spines. A more challenging problem is to tackle rough or corrugated surfaces or, in general, surfaces that have roughness comparable to spine length. Either the feet and toes must have enough "suspension travel" to accommodate the contours of the surface or they must have an additional

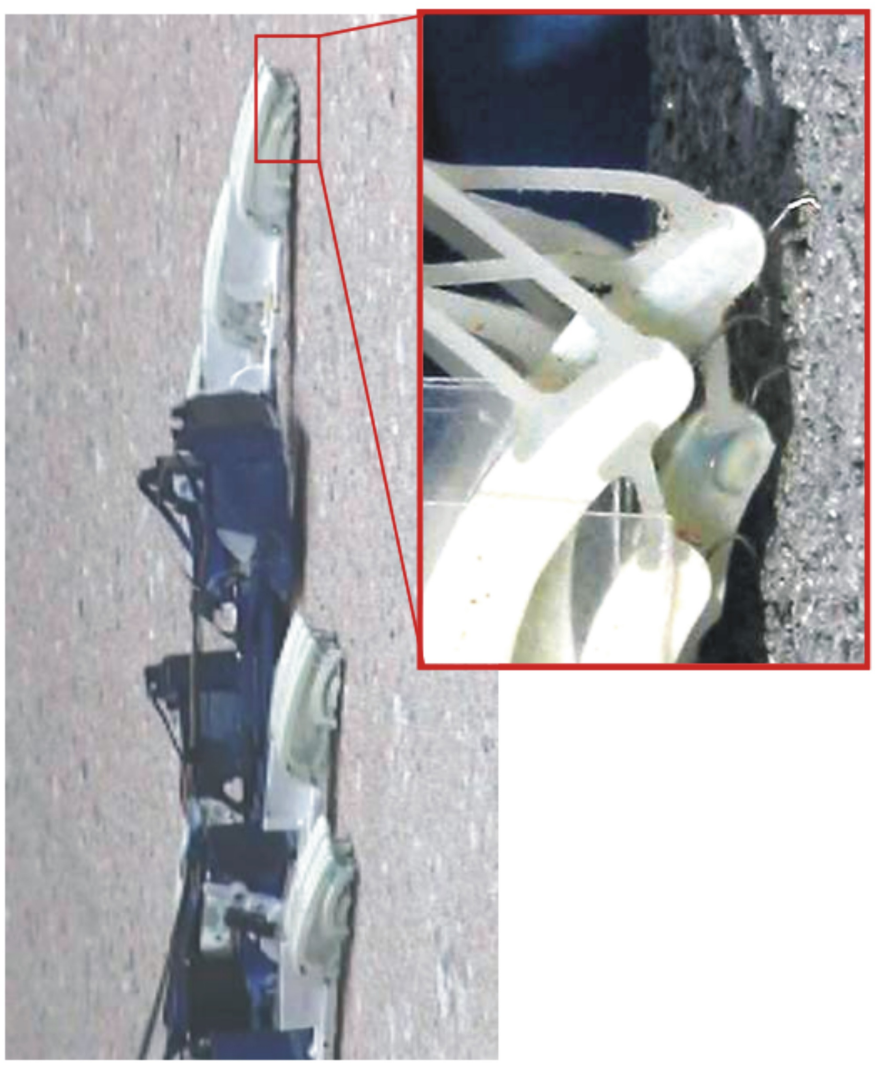

Fig. 7. Picture of upper section of SpinybotII on concrete wall and detailed view of several spines independently engaging asperities on the concrete surface.

active degree of freedom, like the toes of geckos or the tendonactuated tarsus of insect legs. On contoured surfaces it should be possible to exploit internal "grasp" forces, in a manner similar to that used by robots that climb with hand-holds and foot-holds [8], [6], for additional security. The compliant suspension of the spines will become an increasingly difficult design challenge as spines are made smaller: smaller spines necessarily have a smaller clearance, possibly preventing them from reaching deep holes in the surface while maintaining a favorable loading angle.

Another challenging problem is to climb surfaces with much lower roughness than concrete or sandstone, such as polished stone or interior wall panels. The scaling arguments in Sections III and IV should still apply. However, for smooth panels the average asperity radius may be on the order of a few micrometers, requiring spine tip radii of perhaps $1 \mu \mathrm{m}$. These extremely small spines will be over 100 times weaker than the spines on SpinybotII and a large number of them will be required, unless the overall mass of the robot can be reduced correspondingly. Going still smaller, we approach the dimensions of the hairs that are being investigated for synthetic dry adhesives [3], [21], [26], [14]. At the smallest scales, hairs utilizing adhesion have two advantages over microspines or nanospines: they are less sensitive to the local surface normal 
distribution and they are loaded primarily in tension, rather than in bending.

For a given surface, at a small enough length scale, the surface will appear fractal. Beyond that point the number of asperities per unit area will grow as $1 / r^{2}$. Since the spine strength grows as $r^{2}$, we hypothesize that the total weight that can be sustained per unit of surface area using spines is approximately constant. This weight per unit area number will depend on the distribution of surface normal angles, which is related to the surface's fractal dimension.

An interesting question is whether some combination of spines and adhesive hairs will ultimately prove most effective for scaling a variety of hard vertical surfaces. Different surfaces have different distributions of surface normal angles. Spines perform best on surfaces with normal angles close to $90^{\circ}$, while dry adhesives do best on smoother surfaces with normal angles closer to $0^{\circ}$. To be able to climb the widest variety of surfaces, both spines and dry adhesives could be used.

\section{B. Future work}

Future work in modeling spine/surface contacts could benefit from 3-D surface measurement, which would enable more accurate asperity location predictions. For very smooth surfaces, an atomic force microscope or similar instrument may be required to accurately measure the surface heights. For rough surfaces, using a laser interferometer with the surface tilted at an angle could permit measurement of surface normal angles in excess of $90^{\circ}$. For the robot feet, making smaller spines and better suspension systems is the corresponding next step. It is still unknown what the minimum scale is for fabricating and using spines effectively.

A video of SpinybotII climbing various buildings and some close shots of its feet and toes engaging asperities, can be found at http://bdml.stanford.edu/RiSE/Downloads/ . Watching the video closely will reveal several instances in which one foot briefly loses its grip. However, there is enough redundancy and compliance that the robot does not fall. Of course, if the robot encounters a very smooth patch, it either fails to proceed or falls. For greater reliability, we are investigating miniature accelerometers at the toes that will indicate when contact has occurred and whether the foot is stationary or slipping. Other obvious improvements are to increase the climbing speed and to provide additional articulation so that the robot can negiotate vertical/horizontal transitions such as window sills.

\section{APPENDIX: SPINE FAILURE MODES}

\section{APPENDIX}

We have observed that the spine/asperity contacts have three primary failure modes.

1) The spine fails plastically at its base due to tensile stress from bending.

2) The spine deflects elastically such that it slips off the asperity.

3) The asperity fails, typically as a particle becomes unbonded from the surrounding matrix.

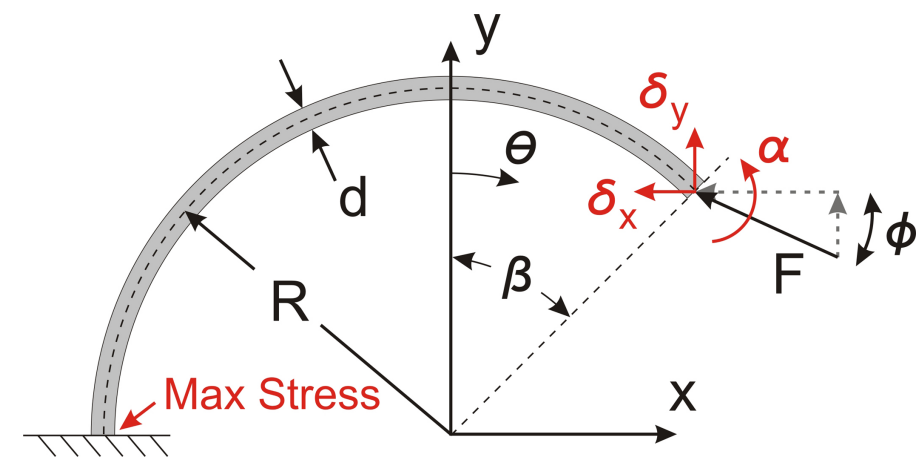

Fig. 8. Curved beam with variables used in spine failure mode analysis.

The first mode of failure is due to the tensile stress at the base of the spine. For a long curved spine, the maximum stress is essentially the same as it would be for a straight cantilever beam [25]:

$$
\sigma_{\max }=\frac{M c}{I}=\frac{32 f l d}{\pi d^{4}} \propto \frac{1}{d^{2}} \quad\left(\text { if } \quad \frac{l}{d}=\text { const }\right)
$$

where

$f=$ force exerted on tip of the spine

$d=$ diameter of cross section of spine

$l=$ equivalent beam length.

The second mode of failure is excessive tip rotation. Here we can apply Castigliano's Theorem to solve for the tip deflections and rotations for a curved spine [25]. Applying a dummy end moment, $M$, and solving for the end rotation, $\alpha$, we obtain:

$$
\begin{aligned}
\alpha= & \partial U / \partial M \\
= & \frac{R^{2}}{2 E I}\left[-2 F_{y}+\left(2 F_{x}+F_{y}(\pi+2 \beta)\right) \cos (\beta)\right. \\
& \left.+\left(-2 F_{y}+F_{x} \pi+2 F_{x} \beta\right) \sin (\beta)\right] \quad \propto \frac{1}{d^{2}} \\
& \text { if } \left.\quad \frac{R}{d}=\text { const. at given } \beta, F_{x} \text { and } F_{y}\right)
\end{aligned}
$$

(see figure 8 ).

The third mode of failure is that the asperity itself may break off. The literature on surface failure or erosion (e.g. [23], [24]) for cementitious materials such as concrete, or rock with hard crystals in a weaker matrix, generally starts with the Hertz stress distribution at the contact [17]. The maximum pressure is at the center of the contact patch:

$$
p_{\max }=3 f / 2 \pi a^{2}=\left(6 f E^{2} / \pi^{3} R^{2}\right)^{1 / 3}
$$

where

$$
\begin{aligned}
a= & (3 f R / 4 E)^{1 / 3} \\
E= & \left(1-\mu_{s}^{2}\right) / E_{s}+\left(1-\mu_{a}^{2}\right) / E_{a} \\
& \left(1 / R=1 / r_{s}+1 / r_{a}\right)
\end{aligned}
$$


and the subscripts $s$ and $a$ refer to the spine tip and asperity, respectively.

The worst case tensile stress is at the periphery of the contact patch:

$$
\sigma_{T}=\left(\left(1-2 \mu_{2}\right) p_{\max }\right) / 3
$$

The actual failure will depend on the local stress state, number of cracks and fracture toughness of the material. However, it will be a function of the maximum tensile stress. Therefore we can write that

$$
f_{\max }=\left[\left(\pi \sigma_{\max } /\left(1-2 \mu_{2}\right)\right)^{3}\left(1 / 2 E^{2}\right)\right] R^{2}
$$

The quantity in square brackets is a constant depending on the materials so that, in the end, the maximum sustainable load is expected to vary as the square of the radii of curvature of the spine tip and asperity.

\section{ACKNOWLEDGMENT}

This work was supported in part by the DARPA Biodynotics Program under Contract NC66001-03-C-8045. Additional support was provided by a DCI fellowship for W. Provancher and a NSF fellowship for A. Asbeck. The authors thank V. Mattoli for his development of the PIC processor program for controlling the RC servos. Thanks are also due to J. Lee for her help in designing and fabricating SpinybotI.

\section{REFERENCES}

[1] http://www.vortexhc.com/vmrp.html.

[2] Discover. 21(9), 2000.

[3] Eduard Arzt, Stanislav Gorb, and Ralph Spolenak. From micro to nano contacts in biological attachment devices" preceedings of national academy of sciences. In Preceedings of National Academy of Sciences, volume 100, pages 10603-10606, 2003.

[4] K. Autumn and A. Peattie. Mechanisms of adhesion in geckos. Integrative and Comparative Biology, 42(6):1081-1090, 2002.

[5] C. Balaguer, A. Gimenez, J.M. Pastor, V.M. Padron, and C. Abderrahim. A climbing autonomous robot for inspection applications in $3 \mathrm{~d}$ complex environments. Robotica, 18:287-297, 2000.

[6] D. Bevly, S. Dubowsky, and C. Mavroidis. A simplified cartesiancomputed torque controller for highly geared systems and its application to an experimental climbing robot. Transactions of the ASME. Journal of Dynamic Systems, Measurement and Control, 122(1):27-32, 2000.

[7] M. Binnard and M.R. Cutkosky. A design by composition approach for layered manufacturing. ASME Transactions, Journal of Mechanical Design, 122(1):91-101, 2000

[8] T. Bretl, S. Rock, and J.C. Latombe. Motion planning for a three-limbed climbing robot in vertical natural terrain. In Proceedings of the IEEE International Conference on Robotics and Automation. Piscataway, NJ, USA : IEEE, 2003.

[9] M. Catmill. Climbing. In M. Hildebrand, D.M. Bramble, K.F. Liem, and D.B. Wake, editors, Functional Vertebrate Morphology, pages 7388. The Belknap Press, Cambridge, 1985.
[10] M. A. Costa and M. R. Cutkosky. Roughness perception of haptically displayed fractal surfaces. In Proceedings of the ASME Dynamic Systems and Control Division, volume 69-2, pages 1073-1079, 2000.

[11] Z.D. Dai, S.N. Gorb, and U. Schwarz. Roughness-dependent friction force of the tarsal claw system in the beetle pachnoda marginata (coleoptera, scarabaeidae). Journal Of Experimental Biology, 205(16):2479-2488, 2002.

[12] S.B. Emerson and D. Diehl. Toe pad morphology and mechanisms of sticking in frogs. Biological Journal of the Linnean Society, 13(3):199216, 1980.

[13] W. Federle, M. Riehle, A.S.G. Curtis, and R.J. Full. An integrative study of insect adhesion: mechanics and wet adhesion of pretarsal pads in ants. Integrative and Comparative Biology, 42(6):1100-1106, 2002.

[14] A. K. Geim, S. V. Dubonos, Grigorieva, K. S. Novoselov, A. A. Zhukov, and S. Yu. Shapoval. Microfabricated adhesive mimicking gecko foothair. Nature Materials, 2:461-463, 2003.

[15] J. A. Greenwood. Contact of rough surfaces. In I. L. Singer and H. M. Pollock, editors, Fundamental of Friction: Macroscopic and Microscopic Processes, pages 37-56. Kluwer Academic Publishers, Dordrecht, The Netherlands, 1992.

[16] J. A. Greenwood. Problem with surface roughness. In I. L. Singer and H. M. Pollock, editors, Fundamental of Friction: Macroscopic and Microscopic Processes, page 5776. Kluwer Academic Publishers, Dordrecht, The Netherlands, 1992.

[17] K.L. Johnson. In Contact Mechanics, pages 72-94. Cambridge University Press, 1985.

[18] A. B. Kesel, A. Martin, and T. Seidl. Adhesion measurements on the attachment devices of the jumping spider evarcha arcuata. The Journal of Experimental Biology, 206:2733-2738, 2003.

[19] G. La Rosa, M. Messina, G. Muscato, and R. Sinatra. A lowcost lightweight climbing robot for the inspection of vertical surfaces Mechatronics, 12(1):71-96, 2002.

[20] R. Lal Tummala, R. Mukherjee, N. Xi, D. Aslam, H. Dulimarta, Jizhong Xiao, M. Minor, and G. Dang. Climbing the walls [robots]. IEEE Robotics and Automation Magazine, 9(4):10-19, 2002.

[21] C. Menon, M. Murphy, and M. Sitti. Gecko inspired surface climbing robots. Proceedings of the IEEE International Conference on Robotics and Biomimetics (ROBIO), 2004.

[22] R. Merz, F.B. Prinz, K. Ramaswami, M. Terk, and L. Weiss. Shape deposition manufacturing. In Proceedings of the Solid Freeform Fabrication Symposium, volume 1-8, August 1994.

[23] A. W. Momber. Damage to rocks and cementitious materials from solid impact. Rock Mechanics and Rock Engineering, 37(1):57-82, 2004.

[24] G.L. Sheldon and I. Finnie. The mechanism of material removal in the erosive cutting of brittle materials. ASME Journal of Engineering for Industry, 88B:393-400, 1966.

[25] C.R. Shigley, J.E.; Mischke. In Standard Handbook of Machine Design (2nd Edition). McGraw-Hill, 1996.

[26] M. Sitti and R.S. Fearing. Synthetic gecko foot-hair micro/nanostructures for future wall-climbing robots. In Proceedings of the IEEE International Conference on Robotics and Automation, volume 1, pages 1164 - 1170. Piscataway, NJ, USA : IEEE, 2003.

[27] Z.L. Xu and P.S. Ma. A wall-climbing robot for labelling scale of oil tank's volume. Robotica, 20:209-212, 2002.

[28] P.A. Zani. The comparative evolution of lizard claw and toe morphology and clinging performance. Journal of Evolutionary Biology, 13:316-325, 2000.

[29] J. Zhu, D. Sun, and S.K. Tso. Development of a tracked climbing robot. Journal Of Intelligent And Robotic Systems, 35(4):427-444, 2002. 